\title{
GENERALIZED DURATION MEASURES IN A RISK IMMUNIZATION SETTING. IMPLEMENTATION OF THE HEATH-JARROW-MORTON MODEL
}

Abstract. The aim of this paper is to set different lower bounds on the change of the expected net cash flow value at time $H>0$ in general term structure models, referring to the studies of Fong and Vasiček (1984), Nawalkha and Chambers (1996), and Balbás and Ibáñez (1998) among others. New immunization strategies are derived with new risk measures: generalized duration and generalized $M$-absolute of Nawalkha and Chambers, and exponential risk measure. Furthermore, examples of specific one-factor HJM models are provided and the problem of immunization is discussed.

1. Introduction. Bondholders are subject to interest risk caused by changes in interest rates. Therefore the problem of bond investment immunization against interest risk is an important issue for bond portfolio managers and researchers. This problem has persisted in the literature since Macaulay's definition of duration (1938), and it was shown independently by Samuelson (1945) and Redington (1952) that if the Macaulay durations of assets and liabilities are equal, the portfolio is protected against a local parallel change in the yield curve. Fisher and Weil (1971) formalize the traditional theory of immunization defining the conditions under which the value of an investment in a bond portfolio is hedged against any parallel shifts in the forward rates. The main result of this theory is that immunization is achieved if the Fisher-Weil duration of the portfolio is equal to the length of the investment horizon (see Rządkowski and Zaremba, 2000). Unfortunately, this traditional approach has serious limitations since it implies

2000 Mathematics Subject Classification: 62P20, 91B28.

Key words and phrases: asset-liability portfolio, immunization, duration, $M$-absolute, one-factor HJM models. 
arbitrage opportunity inconsistent with the rules of modern finance theory. To overcome it, the pioneer work of Fong and Vasiček (1984) indicates a new direction in studying immunization. They propose to determine a lower bound of the changes in a portfolio value which lead to a risk controlling strategy. Nawalkha and Chambers (1996), Balbás and Ibáñez (1998), Balbás et al. (2002), Nawalkha et al. (2003) and Kałuszka and Kondratiuk-Janyska (2004) follow their approach by immunizing a single liability. However, in reality investors have to deal with multiple liabilities (see Hürlimann, 2002) under multiple shocks in the term structure of interest rates (TSIR for short). Moreover, there is a demand for research considering portfolio immunization under stochastic duration in the case of a stream of liability outflows, as over the last decade one of the cornerstones of interest risk management is modeling the stochastic behavior of interest rates. Agca (2002) investigates empirically classical and stochastic durations but does not discuss any portfolio value in connection e.g. with a stochastic duration. To our knowledge the first to study the lower bounds of the net present portfolio value under the above conditions was Gajek (2005) (see also Gajek and Ostaszewski, 2004). He considered the hedging problem at time 0 under random changes of the basic TSIR corresponding to e.g. a supermartingale-like shift factors structure for an insurance company. The derived lower bounds include asset and liability durations as a risk measure.

We do not generalize Gajek's (2005) novel results but inspired by this article we focus on immunizing multiple liabilities, formulating the problem from a different standpoint (see also Kondratiuk-Janyska and Kałuszka, 2006), namely of protected fixed income asset inflows (bonds) and random liability outflows. The aim of this paper is to set different lower bounds on the change of the expected net cash flow value (difference between asset and liability stream) at time $H>0$ which is called a rebalanced time in general term structure models. New immunization strategies are derived with new risk measures like generalized durations (Proposition 3) or generalized $M$-absolute of Nawalkha and Chambers (Proposition 1) and a completely new risk measure (Proposition 2).

The remainder of this paper is organized as follows. Section 2 defines a generalized duration measure and presents examples of stochastic and polynomial durations as particular cases. Section 3 gives the notation and assumptions. Sections 4 presents immunization strategies based on single-risk measure or multiple-risk measure models. Section 5 provides examples of specific one-factor HJM models and discusses the problem of immunization.

2. Generalized duration measures. Duration is unquestionably the most widely used risk measure with a long history. We introduce a generalized duration with respect to a fixed function $\gamma=\gamma(t)$, where particular $\gamma$ 
functions yield well-known durations from different models of interest rate behavior, either deterministic or stochastic. Define

$$
D_{A}(\gamma)=\int_{0}^{T} \int_{0}^{t} \gamma(s) d s d A(t),
$$

where $A(t)$ is an accumulated value of assets described precisely in the next section. In the case $\gamma(t) \equiv 1$, we get the classical duration. When $\gamma(t)=t^{k}, \quad k \geq 1$, we obtain higher order duration risk measures derived from polynomial models; see e.g. Chambers et al. (1988), Prisman and Shores (1988), Rządkowski and Zaremba (2000). On the other hand, generalized durations appear in stochastic models of instantaneous forward rate behavior with appropriate $\gamma$ functions. The most popular arbitrage-free model for describing the term structure is the Heath, Jarrow and Morton (1992) model. This is a very general and popular approach due to its flexibility with respect to the number of random factors used and different volatility structures that can be assumed for different maturity forward rates. When there is one source of randomness, a multi-factor HJM model becomes a one-factor model. Most of the available short rate models are specific cases of one-factor HJM models. Given that short rate models are relatively simple compared to their multi-factor counterparts and that about $90 \%$ of the variation in the yield curve can be explained by only one factor (see Litterman and Scheinkman, 1991), we focus on one-factor HJM models, where the evolution of the instantaneous forward rate on $[0, T]$ is specified by the following stochastic process:

$$
d f(t, T)=\alpha(t, T, \omega) d t+\sigma(t, T, \omega) d W_{t},
$$

where $t \leq T, \alpha(t, T, \omega)$ is the instantaneous forward rate drift function, $\sigma(t, T, \omega)$ is the instantaneous forward rate volatility function and $W_{t}$ is the Brownian motion on a probability space $(\Omega, \mathcal{F}, \mathbb{P})$ equipped with a filtration $\mathbb{F}=\left(\mathcal{F}_{t}\right)_{0 \leq t \leq T}$. Au and Thurston (1995) and Munk (1999) derive the duration measures of certain continuous time one-factor HJM models generalizing the previous result of Cox, Ingersoll and Ross (1979). Denote by $P(t, m)$ the time- $t$ price of a zero-coupon bond maturing at time $m$ and paying one unit $(t \leq m \leq T)$. Write $B(t, T)$ for the time- $t$ price of a bond portfolio with coupons $C_{1}, \ldots, C_{n}$ at dates $t_{1}, \ldots, t_{n}$, where $t \leq t_{i} \leq T$ for $i=1, \ldots, n$. In a classical approach, duration is a measure of the proportional percentage in a bond's price due to shifts in the term structure. Basing on it, Au and Thurston (1995) use the definition of duration at time $t$ which is a special case of $(1)$,

$$
D_{\mathrm{HJM}}=-\frac{\partial B(t, T)}{\partial f(t, t)} / B(t, T),
$$


which yields

$$
D_{\mathrm{HJM}}=\frac{\sum_{i=1}^{n} C_{i} P\left(t, t_{i}\right) \int_{t}^{t_{i}} \sigma(t, s, \omega) d s}{\sigma(t, t, \omega)} / \sum_{i=1}^{n} C_{i} P\left(t, t_{i}\right) .
$$

Some examples are given below for different volatility functions in one-factor HJM models:

- Constant volatility $\sigma(t, T, \omega)=\sigma$ (Merton, 1973; Ho-Lee, 1986),

$$
D_{\mathrm{HJM}}=\frac{\sum_{i=1}^{n} C_{i}\left(t_{i}-t\right) P\left(t, t_{i}\right)}{\sum_{i=1}^{n} C_{i} P\left(t, t_{i}\right)}
$$

- Exponentially decaying volatility $\sigma(t, T, \omega)=\sigma e^{-b(T-t)}($ Vasiček, 1977),

$$
D_{\mathrm{HJM}}=\frac{\sum_{i=1}^{n} C_{i} P\left(t, t_{i}\right)\left(1-e^{-b\left(t_{i}-t\right)}\right)}{b \sum_{i=1}^{n} C_{i} P\left(t, t_{i}\right)} .
$$

- Constant decay volatility $\sigma(t, T, \omega)=\frac{\sigma}{1+T-t}$ (Au and Thurston, 1995),

$$
D_{\mathrm{HJM}}=\frac{\sum_{i=1}^{n} C_{i} P\left(t, t_{i}\right) \ln \left(1+t_{i}-t\right)}{\sum_{i=1}^{n} C_{i} P\left(t, t_{i}\right)}
$$

- Constant maturity $\sigma(t, T, \omega)=\frac{\sigma}{1+T}$ (Au and Thurston, 1995),

$$
D_{\mathrm{HJM}}=\frac{\sum_{i=1}^{n} C_{i} P\left(t, t_{i}\right) \ln \left(\frac{1+t_{i}}{1+t}\right)}{\sum_{i=1}^{n} C_{i} P\left(t, t_{i}\right)}
$$

- Stochastic volatility (Cox, Ingersoll and Ross, 1985),

$$
\begin{aligned}
\int_{t}^{T} \sigma(t, s, \omega) d s & =\frac{2 \sigma \sqrt{f(t, t)} \sinh (\gamma(T-t))}{2 \gamma \cosh (\gamma(T-t))+b \sinh (\gamma(T-t))}, \\
\sigma(t, t, \omega) & =\sigma \sqrt{f(t, t)}, \\
D_{\mathrm{HJM}} & =\sum_{i=1}^{n} \frac{2 C_{i} P\left(t, t_{i}\right) \sinh \left(\gamma\left(t_{i}-t\right)\right)}{2 \gamma \cosh \left(\gamma\left(t_{i}-t\right)\right)+b \sinh \left(\gamma\left(t_{i}-t\right)\right)} / \sum_{i=1}^{n} C_{i} P\left(t, t_{i}\right),
\end{aligned}
$$

where $2 \gamma=\sqrt{b^{2}+2 \sigma^{2}}$ and $b, \sigma>0, \sinh (x)=\frac{e^{x}-e^{-x}}{2}, \cosh (x)=\frac{e^{x}+e^{-x}}{2}$.

Although a class of duration measures for the HJM interest rate model has been constructed (Cox, Ingersoll and Ross, 1979; Au and Thurston, 1995; Munk, 1999), there are no studies determining the lower bound of the change in a bond portfolio value in this model. Therefore, the aim of this paper is to introduce arbitrage-free models setting different lower bounds on the change of the expected net cash flow value (difference between asset and liability stream) where a generalized duration is an immunization measure. 
3. Preliminary notations. Denote by $[0, T]$ the time interval with $t=0$ the present moment, and let $H$ be an investor planning horizon, $0<H<T$, when the portfolio is rebalanced. The portfolio consists of bond inflows $A_{t} \geq 0$ occurring at fixed time $t \leq T\left(t=t_{1}, \ldots, t_{d}\right)$ to cover multiple liabilities $L_{t}$ due at dates $t \leq T\left(t=t_{1}, \ldots, t_{d}\right)$, where $t_{d}=T$. This is a typical situation e.g. when an insurance company has to discharge its random liabilities and invests the money by acquiring an immunized bond portfolio. Denote the set of available bonds by $\mathcal{A}$. Generally, this is an arbitrary subset of $[0, \infty)^{d}$ that might be nonconvex since we do not assume that the market is complete and bonds are infinitely divisible. Additionally, we assume that liabilities are nonnegative random variables. Consequently, $N_{t}=A_{t}-L_{t}$ is the net cash flow at time $t$. Let $f(t, s)$ be an instantaneous forward rate over the time interval $[t, s]$; investing 1 at time $t$ in a zero coupon-bond we get $\exp \left(\int_{t}^{s} f(t, u) d u\right)$ at time $s$. The set of instantaneous forward rates $\{f(t, s)$ : $0<t \leq s\}$ determines a random term structure of interest rates. Hence

- $a_{t}=A_{t} \exp \left(\int_{t}^{H} f(0, u) d u\right)$ is the time- $H$ value of $A_{t}$,

- $l_{t}=L_{t} \exp \left(\int_{t}^{H} f(0, u) d u\right)$ is the time- $H$ value of $L_{t}$,

- $n_{t}=a_{t}-l_{t}$ is the time- $H$ value of net worth,

- $A(t)=\sum_{s \leq t} a_{s}$ is an accumulated value of assets,

- $L(t)=\sum_{s \leq t} l_{s}$ is an accumulated value of liabilities,

- $N(t)=A(\bar{t})-L(t)$

- $V(0)=\mathbf{E} \sum_{t} n_{t}=\mathbf{E} N(T)$ is the time- $H$ average value of the portfolio of asset and liability flows if forward rates equal future spot rates.

A decision problem for an investor is to design a stream of bonds to cover the stream of liabilities. If among available bonds there are such that $N_{t}=0$ for all $t$, then the portfolio is immunized. In reality, the market is incomplete, which excludes an ideal adjustment of assets to liabilities. An investor constructing a bond portfolio meets two kinds of risks: reinvestment and price. The first one is connected with the way of reinvesting coupons paid before the investment horizon. The other appears by pricing bonds before their expiry dates. Since the portfolio value at time $H$ depends on the reinvestment strategy, we require the following open-loop strategy:

(a) If $t<H$ then the value of $N_{t}$ at time $H$ is equal to

$$
N_{t} \exp \left(\int_{t}^{H} f(t, s) d s\right) .
$$

That means that if $N_{t}=A_{t}-L_{t}>0$ for $0<t<H$, the investor purchases $(H-t)$-year strip bonds. Otherwise, he sells short $(H-t)$ year strip bonds. 
(b) If $t>H$, the value of $N_{t}$ at $H$ equals

$$
N_{t} \exp \left(-\int_{H}^{t} f(H, s) d s\right)=N_{t} \exp \left(\int_{t}^{H} f(H, s) d s\right),
$$

which means that at time $H$ the portfolio priced according to the TSIR is sold by the investor.

As a consequence, the value of the net cash flow at $H$ equals

$$
\sum_{t} N_{t} \exp \left(\int_{t}^{H} f(t \wedge H, s) d s\right)=\sum_{t} n_{t} \exp (k(t)),
$$

where

$$
k(t)=\int_{t}^{H}[f(t \wedge H, s)-f(0, s)] d s
$$

is a shock in the instantaneous forward rate and $a \wedge b=\min (a, b)$. From the investor's standpoint, the average time- $H$ value of $N_{t}$ under the scenario (a)-(b) is given by

$$
V(k)=\mathbf{E}\left(\int_{0}^{T} \exp (k(t)) d N(t)\right) .
$$

The classical immunization problem is to find a portfolio such that $V(k) \geq$ $V(0)$ for all $k \in \mathcal{K}$, where $\mathcal{K}$ stands for a feasible class of shocks. Our aim is to find a lower bound on $\inf _{k \in \mathcal{K}} V(k)$ which depends only on bond portfolio proportions. Next, we select at $t=0$ a portfolio among available bonds on the market such that this lower bound is maximal.

\section{Risk measure models}

M-absolute as a risk measure. The linear cash flow dispersion measure, called the $M$-absolute, defined by Nawalkha and Chambers (1996),

$$
M_{\mathrm{NCh}}=\frac{\int_{0}^{T}|t-H| d A(t)}{\int_{0}^{T} d A(t)},
$$

is an immunization risk measure designed to build immunized bond portfolios in the case of a single liability. In the case of multiple liabilities, we define the generalized M-absolute by

$$
M=\int_{0}^{T}|A(t)-A(T)+\mathbf{E}(L(T)-L(t))| d t .
$$

It is easily seen that $M=A(T) M_{\mathrm{NCh}}$ in the case of a single nonrandom liability at time $H$. 
The following assumptions will be needed throughout the paper:

A1. A random variable $l_{t}$ is independent of the TSIR for every $t>0$.

A2. $\left(\mathbf{E} e^{k(t)}\right)^{\prime}$ is continuous on $[0, T]$.

Define the generalized durations of assets and liabilities

$$
D_{A}(\gamma)=\int_{0}^{T} \int_{0}^{t} \gamma(s) d s d A(t), \quad D_{L}(\gamma)=\mathbf{E} \int_{0}^{T} \int_{0}^{t} \gamma(s) d s d L(t),
$$

respectively, where $\gamma=\gamma(t)$ is a fixed function.

Proposition 1. Under assumptions $\mathbf{A 1 - A 2 , ~ a ~ l o w e r ~ b o u n d ~ o n ~ t h e ~ p o s t - ~}$ shifts change in the value of the net cash flow at $H$ is given by

$$
\inf _{k \in \mathcal{K}_{1}} V(k)-V(0) \geq-k M+D_{A}(\gamma)-D_{L}(\gamma),
$$

where $\mathcal{K}_{1}=\left\{k(\cdot):\left|\left(\mathbf{E} e^{k(t)}\right)^{\prime}-\gamma(t)\right| \leq k\right.$ for all $\left.t \in[0, T]\right\}, k$ being a nonnegative number.

Proof. From assumption A1, we get

$$
\begin{aligned}
V(k)= & \sum_{t} \mathbf{E}\left[n_{t} e^{k(t)}\right]=\int_{0}^{T} \mathbf{E} e^{k(t)} d \mathbf{E} N(t) \\
= & \mathbf{E} e^{k(T)} \mathbf{E} N(T)-\int_{0}^{T} \mathbf{E} N(t)\left(\mathbf{E} e^{k(t)}\right)^{\prime} d t \\
= & \int_{0}^{T}(\mathbf{E} N(T)-\mathbf{E} N(t))\left(\mathbf{E} e^{k(t)}\right)^{\prime} d t+\mathbf{E} N(T) \\
= & \int_{0}^{T}(\mathbf{E} N(T)-\mathbf{E} N(t))\left(\left(\mathbf{E} e^{k(t)}\right)^{\prime}-\gamma(t)\right) d t \\
& +\int_{0}^{T} \gamma(t)(\mathbf{E} N(T)-\mathbf{E} N(t)) d t+\mathbf{E} N(T) .
\end{aligned}
$$

Since $\mathbf{E} N(T)=V(0)$, for all $k(\cdot) \in \mathcal{K}_{1}$ we have

$$
\begin{aligned}
V(k)-V(0) \geq & -k \int_{0}^{T}|\mathbf{E} N(t)-\mathbf{E} N(T)| d t+\left.\int_{0}^{t} \gamma(s) d s(\mathbf{E} N(T)-\mathbf{E} N(t))\right|_{0} ^{T} \\
& +\int_{0}^{T} \int_{0}^{t} \gamma(s) d s d \mathbf{E} N(t),
\end{aligned}
$$

as desired. 
As a corollary of Proposition 1 we get the following immunization strategy:

$$
\begin{aligned}
& \min _{\left(A_{t}\right) \in \mathcal{A}} \int_{0}^{T}|A(T)-A(t)+\mathbf{E}(L(t)-L(T))| d t \\
& \text { subject to } D_{A}(\gamma)-D_{L}(\gamma)=d,
\end{aligned}
$$

where $d$ is a fixed nonnegative value of a duration gap.

EXAMPLE 1. Suppose that three kinds of zero-coupon bonds are available on the market. The face value of the bond at the maturity date $t=1,2,4$ is $B_{t}$ and the investor is to discharge fixed liabilities $P_{t}$ at $t=3,5$. Take $T=5$ and let the planning horizon be $H=3$. The time- 3 value of $B_{t}$ and $P_{t}$ is denoted by $b_{t}$ and $p_{t}$, respectively. Consider the situation when the expenditure-income plan is such that $N(5)=0, \gamma(t) \equiv \gamma$ and $d$ is a nonnegative real number. Denoting by $x_{t}$ the amount of purchased $t$-year bond units, the immunization problem should be solved according to the model:

$$
\begin{gathered}
\min _{\left(x_{t}\right)} \int_{0}^{T}\left|\sum_{s \leq t}\left(x_{s} b_{s}-p_{s}\right)\right| d t \\
\text { subject to } \quad \sum_{t} x_{t} b_{t}=\sum_{t} p_{t}, \quad \gamma \sum_{t} t\left(x_{t} b_{t}-p_{t}\right)=d, \\
b_{t} \geq 0 \text { for } t=1,2,4 .
\end{gathered}
$$

Solving problem (5) we obtain

$$
x_{1}=0, \quad x_{2}=\frac{1}{2 b_{2}}\left(p_{3}-p_{5}-\frac{d}{\gamma}\right), \quad x_{4}=\frac{1}{2 b_{4}}\left(p_{3}+3 p_{5}+\frac{d}{\gamma}\right)
$$

under the condition $p_{3} \geq p_{5}+d / \gamma$. If $p_{3}<p_{5}+d / \gamma$, then the set of constraints is empty. If we take $\gamma(t)=\gamma t$, we get

$$
x_{1}=0, \quad x_{2}=\frac{1}{b_{2}}\left(\frac{7}{12} p_{3}+\frac{3}{4} p_{5}-\frac{1}{6} \frac{d}{\gamma}\right), \quad x_{4}=\frac{1}{b_{4}}\left(\frac{5}{12} p_{3}+\frac{1}{4} p_{5}+\frac{1}{6} \frac{d}{\gamma}\right)
$$

when $p_{3} \geq \frac{2}{7} \frac{d}{\gamma}-\frac{9}{7} p_{5}$. Otherwise, the set of constraints is empty.

Exponential risk measure. In this subsection we present a lower bound on the change of the expected net cash flow value based on an exponential risk measure. Let us introduce an entropy function

$$
H(f)=\int_{0}^{T} f(t) \ln f(t) d t-\int_{0}^{T} f(t) d t \ln \left(\int_{0}^{T} f(t) d t\right) .
$$


Proposition 2. Let assumptions A1-A2 hold. Then

$$
\begin{aligned}
\inf _{k \in \mathcal{K}_{2}} V(k)-V(0) \geq & -k_{1}-k_{2} \ln \left(\int_{0}^{T} e^{|\mathbf{E}(N(T)-N(t))|} d t\right) \\
& +D_{A}(\gamma)-D_{L}(\gamma),
\end{aligned}
$$

where $\mathcal{K}_{2}=\left\{k(\cdot): H\left(\left|\left(\mathbf{E} e^{k(\cdot)}\right)^{\prime}-\gamma(\cdot)\right|\right) \leq k_{1}, \int_{0}^{T}\left|\left(\mathbf{E} e^{k(t)}\right)^{\prime}-\gamma(t)\right| d t \leq k_{2}\right\}$, and $k_{1}$ and $k_{2}$ are nonnegative numbers.

Proof. By the proof of Proposition 1 we get

$$
\begin{aligned}
V(k)-V(0)= & \int_{0}^{T} \mathbf{E}(N(T)-N(t))\left(\mathbf{E} e^{k(t)}\right)^{\prime} d t \\
\geq & -\int_{0}^{T}|\mathbf{E}(N(T)-N(t))|\left|\left(\mathbf{E} e^{k(t)}\right)^{\prime}-\gamma(t)\right| d t \\
& +\int_{0}^{T} \gamma(t)(\mathbf{E}(N(T)-N(t))) d t .
\end{aligned}
$$

Applying the Young inequality we obtain

$$
\begin{aligned}
V(k)-V(0) \geq & -H\left(\left|\left(\mathbf{E} e^{k(\cdot)}\right)^{\prime}-\gamma(\cdot)\right|\right) \\
& -\int_{0}^{T}\left|\left(\mathbf{E} e^{k(t)}\right)^{\prime}-\gamma(t)\right| d t \ln \left(\int_{0}^{T} e^{|\mathbf{E}(N(T)-N(t))|} d t\right) \\
& +\int_{0}^{T} \gamma(t)(\mathbf{E} N(T)-\mathbf{E} N(t)) d t,
\end{aligned}
$$

which completes the proof of (6).

Inequality (6) implies the following immunization problem:

$$
\begin{aligned}
& \text { find a portfolio which minimizes } \int_{0}^{T} \exp |\mathbf{E}(N(T)-N(t))| d t \\
& \text { subject to } D_{A}(\gamma)-D_{L}(\gamma)=d,
\end{aligned}
$$

where $d$ is a fixed nonnegative value of a duration gap.

ExAmple 2. Under the assumptions as in Example 1 in the case $\gamma(t)$ $\equiv \gamma$, strategy (7) leads to the following optimization problem:

$$
\begin{array}{ll}
\min _{\left(x_{t}\right)} & \int_{0}^{T} \exp \left|\sum_{s \leq t}\left(x_{s} b_{s}-p_{s}\right)\right| d t \\
\text { subject to } & \sum_{t} x_{t} b_{t}=\sum_{t} p_{t}, \quad \gamma \sum_{t} t\left(x_{t} b_{t}-p_{t}\right)=d, \\
& b_{t} \geq 0 \text { for } t=1,2,4 .
\end{array}
$$


Solving problem (8) we obtain the same results as in the previous example, i.e. $x_{1}=0, x_{2}=\frac{1}{2 b_{2}}\left(p_{3}-p_{5}-d / \gamma\right), x_{4}=\frac{1}{2 b_{4}}\left(p_{3}+3 p_{5}+d / \gamma\right)$ under the condition $p_{3} \geq p_{5}+d / \gamma$. If $p_{3}<p_{5}+d / \gamma$, then the set of constraints is empty.

Generalized duration as a risk measure. An appropriate strategy would be to hold a portfolio of assets whose schedule of cash flow covers the pattern of liabilities under a constant TSIR. Thus, it is worth considering immunization among portfolios satisfying a weak version of the Axiom of Solvency (Gajek, 2005):

$$
\mathcal{A}_{0}=\{A(\cdot): A(t) \geq \mathbf{E}(N(T)+L(t)) \text { for all } t \in[0, T]\} .
$$

Proposition 3. Under assumptions $\mathbf{A} \mathbf{1}-\mathbf{A 2}$ and for all $A(\cdot) \in \mathcal{A}_{0}$,

$$
\inf _{k \in \mathcal{K}_{3}} V(k)-V(0) \geq D_{A}(\gamma)-D_{L}(\gamma)
$$

where $\mathcal{K}_{3}=\left\{k(\cdot):\left(\mathbf{E} e^{k(t)}\right)^{\prime} \leq \gamma(t)\right.$ for all $\left.t \in[0, T]\right\}$.

Proof. By the proof of Proposition 1 we get

$$
\begin{aligned}
V(k)-V(0) & =\int_{0}^{T}(\mathbf{E} N(T)-\mathbf{E} N(t))\left(\mathbf{E} e^{k(t)}\right)^{\prime} d t \\
& \geq \int_{0}^{T}(\mathbf{E} N(T)-\mathbf{E} N(t)) \gamma(t) d t,
\end{aligned}
$$

which completes the proof.

As a consequence of Proposition 3 we obtain the strategy:

find a portfolio which maximizes $D_{A}(\gamma)-D_{L}(\gamma)$

subject to $A(t) \geq \mathbf{E}(N(T)+L(t))$ for all $t \in[0, T]$.

5. Nonstandard selection of $\gamma$ functions in stochastic models. To apply Propositions $1-3$ we need a $\gamma$ function. Obviously, one may take $\gamma(t)=1$ or $\gamma(t)=2 t$ for $0 \leq t \leq T$, which gives the well-known traditional duration or convexity, respectively. But such a choice is justified only if we cannot model the TSIR because of the lack of data or an unexpected event. In the case when we use stochastic models of the TSIR, the main question is what a shock concerns. We will say that a shock appears when a model is incorrectly fitted to the reality or when the assumed model parameters differ from real ones. According to the above remark we require in Proposition 1 this deviation to be within a band of width $k$. Hence and by the definition of $\mathcal{K}_{1}$ we conclude that

1. If $k=0$, then a model is perfectly fitted and $\left(\mathbf{E} e^{k(t)}\right)^{\prime}=\gamma(t)$ for $0 \leq t \leq T$. 
2. If $k>0$, the model under the assumed parameters is unadjusted and $k$ measures the deviation of $\gamma(\cdot)$ from the unknown real $\left(\mathbf{E} e^{k(\cdot)}\right)^{\prime}$.

Therefore we propose to take $\gamma$ such that

$$
\left(\mathbf{E} e^{k(t)}\right)^{\prime}=\gamma(t) \quad \text { for } 0 \leq t \leq T .
$$

In particular, in the HJM model we have the following examples where $\left(\mathbf{E} e^{k(t)}\right)^{\prime}$ is not a constant function. In the Merton model, where $f(t, t)=$ $r_{0}+a t+\sigma W_{t}^{*}$, and $r_{0}, a, \sigma$ are positive constants, we have

$$
\begin{aligned}
& \left(\mathbf{E} e^{k(t)}\right)^{\prime} \\
& =\left\{\begin{array}{r}
\frac{\sigma^{2}}{2}\left(2(t-H)^{2}-2 t(t-H)-t^{2}\right) \exp \left(\frac{\sigma^{2}}{6}\left((t-H)^{3}+3 t(t-H)^{2}+H^{3}-t^{3}\right)\right) \\
\text { for } t \leq H, \\
\frac{\sigma^{2}}{2}\left((t-H)^{2}+2 H(t-H)-t^{2}\right) \exp \left(\frac{\sigma^{2}}{6}\left((t-H)^{3}+3 H(t-H)^{2}+H^{3}-t^{3}\right)\right) \\
\text { for } t>H .
\end{array}\right.
\end{aligned}
$$

In the Vasiček model described by $d f(t, t)=(a-b f(t, t)) d t+\sigma d W_{t}^{*}$ where $a, b, \sigma$ are positive constants, we get

$$
\begin{aligned}
& \left(\mathbf{E} e^{k(t)}\right)^{\prime} \\
& = \begin{cases}\frac{\sigma^{2}}{2 b^{2}}\left(-3 e^{-b(H-t)}+2 e^{-2 b(H-t)}-e^{-b(H+t)}+2 e^{-b t}\right) & \\
\times \exp \left(\frac{\sigma^{2}}{2 b^{3}}\left(-3 e^{-b(H-t)}+e^{-2 b(H-t)}+e^{-b(H+t)}+2\left(e^{-b H}-e^{-b t}\right)-e^{-2 b H}+2\right)\right) & \text { for } t \leq H, \\
\frac{\sigma^{2}}{2 b^{2}}\left(-e^{-b(t-H)}-e^{-b(H+t)}+2 e^{-b t}\right) & \text { for } t>H . \\
\times \exp \left(\frac{\sigma^{2}}{2 b^{3}}\left(e^{-b(t-H)}+e^{-b(H+t)}+2\left(e^{-b H}-e^{-b t}\right)-e^{-2 b H}-1\right)\right)\end{cases}
\end{aligned}
$$

In the above models $W^{*}$ is a one-dimensional standard Brownian motion under the spot martingale measure $\mathbb{P}^{*}$. This is our suggestion of a $\gamma$ selection different from standard functions derived in stochastic models (see Au and Thurston, 1995; Munk, 1999). The comparison of their effectiveness demands a huge empirical research, which exceeds the scope of this paper.

\section{References}

S. Agca (2002), The performance of alternative interest rate risk measures and immunization strategies under a Heath-Jarrow-Morton framework, part of dissertation work available at http://scholar.lib.vt.edu/theses/available/etd-04062002-025744/unrestricted/SenayAgcaDissertation.pdf.

K. T. Au and D. C. Thurston (1995), A new class of duration measures, Economics Lett. $47,371-375$.

A. Balbás and A. Ibáñez (1998), When can you immunize a bond portfolio?, J. Banking Finance 22, 1571-1594. 
A. Balbás, A. Ibáñez and S. López (2002), Dispersion measures as immunization risk measures, ibid. 26, 1229-1244.

D. R. Chambers, W. T. Carleton and R. W. McEnally (1988), Immunizing default-free bond portfolios with duration vector, J. Financial Quantitative Anal. 23, 89-1104.

J. C. Cox, J. E. Ingersoll and S. A. Ross (1979), Duration and the measurement of basis risk, J. Business 52, 51-61.

J. C. Cox, J. E. Ingersoll and S. A. Ross (1985), A theory of the term structure of interest rates, Econometrica 53, 385-407.

L. Fisher and R. L. Weil (1971), Coping with risk of interest rate fluctuations: returns to bondholders from naive and optimal strategies, J. Business 44, 408-431.

H. G. Fong and O. A. Vasiček (1984), A risk minimizing strategy for portfolio immunization, J. Finance 39, 1541-1546.

L. Gajek (2005), Axiom of solvency and portfolio immunization under random interest rates, Insurance Math. Economics 36, 317-328.

L. Gajek and K. Ostaszewski (2004), Financial Risk Management for Pension Plans, Elsevier, Amsterdam.

D. Heath, R. Jarrow and A. J. Morton (1992), Bond pricing and the term structure of interest rates: a new methodology for contingent claims valuation, Econometrica 60, $77-105$.

T. S. Y. Ho and S. Lee (1986), Term structure movements and pricing interest rate contingent claims, J. Finance 41, 1011-1029.

W. Hürlimann (2002), On immunization, stop-loss order and the maximum Shiu measure, Insurance Math. Economics 31, 315-325.

M. Kałuszka and A. Kondratiuk-Janyska (2004), On risk minimizing strategies for defaultfree bond portfolio immunization, Appl. Math. (Warsaw) 31, 259-272.

A. Kondratiuk-Janyska and M. Kałuszka (2006), Assets/liabilities portfolio immunization as an optimization problem, Control Cybernet., to appear.

R. Litterman and J. Scheinkman (1991), Common factors affecting bond returns, J. Fixed Income 3, 54-61.

F. Macaulay (1938), Some theoretical problems suggested by the movement of interest rates, bond yields, and stock prices in the US since 1856, National Bureau of Economic Research, New York.

C. Munk (1999), Stochastic duration and fast coupon bond option pricing in multi-factor models, Rev. Derivatives Research 3, 157-181.

S. K. Nawalkha and D. R. Chambers (1996), An improved immunization strategy: $M$ absolute, Financial Analysts J. 52, 69-76.

S. K. Nawalkha, G. M. Soto and J. Zhang (2003), Generalized M-vector models for hedging interest rate risk, J. Banking Finance 27, 1581-1604.

E. Z. Prisman and M. R. Shores (1988), Duration measures for specific term structure estimations and applications to bond portfolio immunization, ibid. 12, 493-504.

F. M. Redington (1952), Review of the principle of life-office valuations, J. Instit. Actuaries $18,286-340$.

G. Rządkowski and L. S. Zaremba (2000), New formulas for immunizing durations, J. Derivatives, 28-36.

P. A. Samuelson (1945), The effects of interest rates increases on the banking system, Amer. Economic Rev. 35, 16-27. 
F. Vasiček (1977), An equilibrium characterization of the term structure, J. Financial Economics 5, 177-188.

Alina Kondratiuk-Janyska

Center of Mathematics and Physics

Technical University of Łódź

Al. Politechniki 11

90-924 Łódź, Poland

and

Institute of Mathematics

Technical University of Łódź

Wólczańska 215

93-005 Łódź, Poland

E-mail: akondrat@p.lodz.pl
Marek Kałuszka

Institute of Mathematics Technical University of Łódź

Wólczańska 215

93-005 Łódź, Poland

E-mail: kaluszka@p.lodz.pl

Received on 1.3.2006;

revised version on 5.5.2006 
